\title{
Numerical analysis of planar multimode optical sensor with active nanolayer
}

\author{
MAREK BŁAHUT \\ Optoelectronics Department, Silesian University of Technology, \\ ul. Krzywoustego 2, 44-100 Gliwice, Poland; marek.blahut@polsl.pl
}

\begin{abstract}
In the paper, numerical studies of the model of an optical sensor, based on interference of modes in a planar one-dimensional step-index configuration, are presented. Calculations are performed using the method of mode field analysis. The structure consists of the single-mode input waveguide, the multimode waveguide that guides only a few modes and the single-mode output waveguide. The structure is covered by a nanometer active sensor layer of a high refractive index, which changes its optical properties in contact with the measured external surrounding. The refractive index variation of an active sensor layer affects the modal properties of the multimode waveguide and the output optical field distribution. By the proper selection of the active layer, the considered configuration can be used for gas detection.
\end{abstract}

Keywords: multimode interference, optical sensors, planar waveguides.

\section{Introduction}

The interference of modes in multimode waveguides depends on their propagation constants difference, which can be changed by variations of the propagation conditions of light. This effect can be used in multimode optical sensor technology. In most cases, the multimode interference (MMI) section is covered with a layer whose optical parameters change when they are exposed to a measured medium or physical fields. There are different possibilities of material selection of the cover. It can be a simple dielectric medium $[1,2]$ which changes its refractive index. Specific modification of this configuration uses the additional thin buffer layer of a high refractive index placed between the dielectric sensing layer and the gradient core of the MMI section. The buffer layer aims to focus the field energy close to the sensor layer and thus to increase the impact of parameters of the cladding on the signal [ $\underline{3}]$. The MMI structures used in sensors technology can be step-index [1] or gradient index made by ion exchange in glass [4]. The operation characteristics of the sensors depend on the geometry and size of MMI structures. Generally, 2D configurations of waveguides are used which are single-mode in the direction perpendicular to the substrate and multimode in a parallel direction.

In this paper, the model of a simple optical sensor in a one-dimensional step-index configuration has been proposed, involving vertical interference of modes. The exam- 
ined structure consists of single-mode input and output waveguides and multimode waveguide between them, covered by a nanometer active layer of a high refractive index. The refractive index of the active sensor layer, substantially higher than the refractive index of MMI section, is the reason for the concentration of wave propagation energy in the sensor layer area and its vicinity. The impact of geometrical and optical parameters of the optical structure on the operating characteristics of the sensor is discussed. The material configuration refers to the SU-8 polymer waveguide on $\mathrm{SiO}_{2}$ substrate. SU-8 is suitable as a waveguide layer since it possesses a low optical absorption and the fabrication process is fast and easy technologically $[\underline{5}, \underline{6}]$. However, results obtained can be easily adapted for other step-index material configurations with similar refractive indices, e.g., for $\mathrm{SiON}$ on $\mathrm{SiO}_{2}$ substrate [7]].

\section{Configuration of examined optical sensor}

Optical configuration of the examined optical structure is shown in Fig. 1. It consists of single-mode input and output waveguides and MMI section. The MMI section is covered by a nanometer active sensing layer, which changes its optical properties in contact with the measured external surrounding. The refractive index variation of an active sensor layer affects the modal properties of the multimode waveguide and the output optical field distribution.

Single-mode output waveguide registers the field variations. Material parameters, shown in Fig. 1, concern the polymer SU-8 waveguide (refractive index $n_{\mathrm{MMI}}=1.595$ [ $[\underline{5}$ ) on $\mathrm{SiO}_{2}$ substrate $\left(n_{\mathrm{b}}=1.466\right)$ for the wavelength $\lambda=633 \mathrm{~nm}$. The thickness of the input waveguide for a single-mode operation amounts to $400 \mathrm{~nm}$, and the thickness of a few-mode multimode section is $800 \mathrm{~nm}$. It is assumed that the length of single-mode input and single-mode output waveguides, as well as the length $L_{\mathrm{MMI}}$ of analyzed MMI section, is equal to $1 \mathrm{~cm}$.

The presented configuration is addressed to the gas detection. Metal oxides $\left(\mathrm{SnO}_{2}\right.$, $\mathrm{ZnO}, \mathrm{TiO}_{2}$, and $\mathrm{WO}_{3}$ ) are generally used in gas sensors as sensing layers []] These materials are characterized by high sensitivity to $\mathrm{CO}, \mathrm{H}_{2}, \mathrm{NO}_{x}, \mathrm{CO}_{2}, \mathrm{O}_{3}$, and hydrocarbons. Their refractive indices are much higher than an SU-8 refractive index.

The number of modes of the multimode waveguide and their wave functions distribution depend on the active layer depth and its refractive index. It is assumed that

a

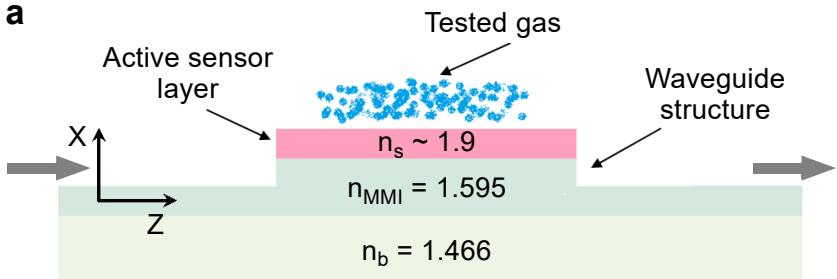

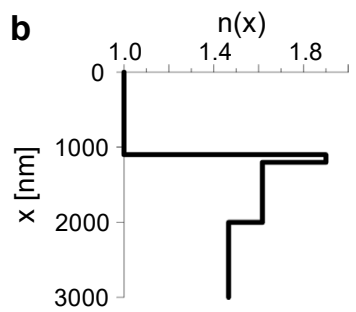

Fig. 1. The geometry of one-dimensional MMI structure in step-index configuration with the active sensor layer (a) and refractive index distribution for the multimode waveguide region (b). 
the refractive index of the layer is about 1.9, which corresponds to $\mathrm{WO}_{3}$ material [ $\underline{9}$ ]. $\mathrm{WO}_{3}$ nanolayer seems a promising material, which provides detection of several gas types, i.e., $\mathrm{NO}_{2}, \mathrm{H}_{2}, \mathrm{NH}_{3}[\underline{10}, \underline{11}]$. The fundamental technological problem to solve in designing of MMI interference sensor with $\mathrm{WO}_{3}$ nanolayer is the selection of the proper nanolayer thickness. It is assumed in the work that its depth changes in the limit from 0 to $200 \mathrm{~nm}$.

It is important for the practical realization of the proposed sensing structure to establish the method of its excitation at the input and signal detection at the output. The simplest way is to use prism couplers at the input and the output of the single-mode waveguide. This is a good solution for the testing. Finally, grating couplers can be used, made at the input and the end of SU-8 single mode waveguides. Technology of such structures, on the base of SU-8 platform, is described in [12].

\section{Modal fields analysis}

The modal field analysis is a useful method for determining the operation principle of examined optical structure. It is convenient in a planar configuration, especially when MMI section guides only a few modes. The wave function $\varphi_{0}(x)$ of the single-mode waveguide describes the field $E_{\text {in }}$ at the input of MMI section,

$$
E_{\text {in }}=\varphi_{0}(x)
$$

This field introduced to the interference section is decomposed into the wave fields of all modes of multimode waveguide:

$$
E_{\mathrm{M}}(x, 0)=\sum_{l=0}^{N-1} c_{l} \varphi_{l}^{\mathrm{M}}(x)
$$

where $\varphi_{l}^{\mathrm{M}}(x)$ are the wave functions of MMI section of an order $l$ with propagation constants $\beta_{l}$ and excitation coefficients $c_{l}$ are defined by overlap integrals:

$$
c_{l}=\int_{-\infty}^{+\infty} \varphi_{0}^{*}(x) \varphi_{l}^{\mathrm{M}}(x) \mathrm{d} x
$$

where $\varphi_{0}^{*}(x)$ is a conjugated wave function, with assumed normalized conditions for $\varphi_{l}^{\mathrm{M}}(x)$ :

$$
\int_{-\infty}^{+\infty}\left|\varphi_{l}^{\mathrm{M}}(x)\right|^{2} \mathrm{~d} x=1
$$

Figure 2 presents the numerically calculated wave functions of MMI sections of the thickness of $800 \mathrm{~nm}$ for different active layer depths $d$. The $\varphi_{2}^{\mathrm{M}}$ wave function appears for the active layer depth near $165 \mathrm{~nm}$. The wave functions distributions of the multimode waveguide are compared with the wave function $\varphi_{0}$ of a single-mode input/ 

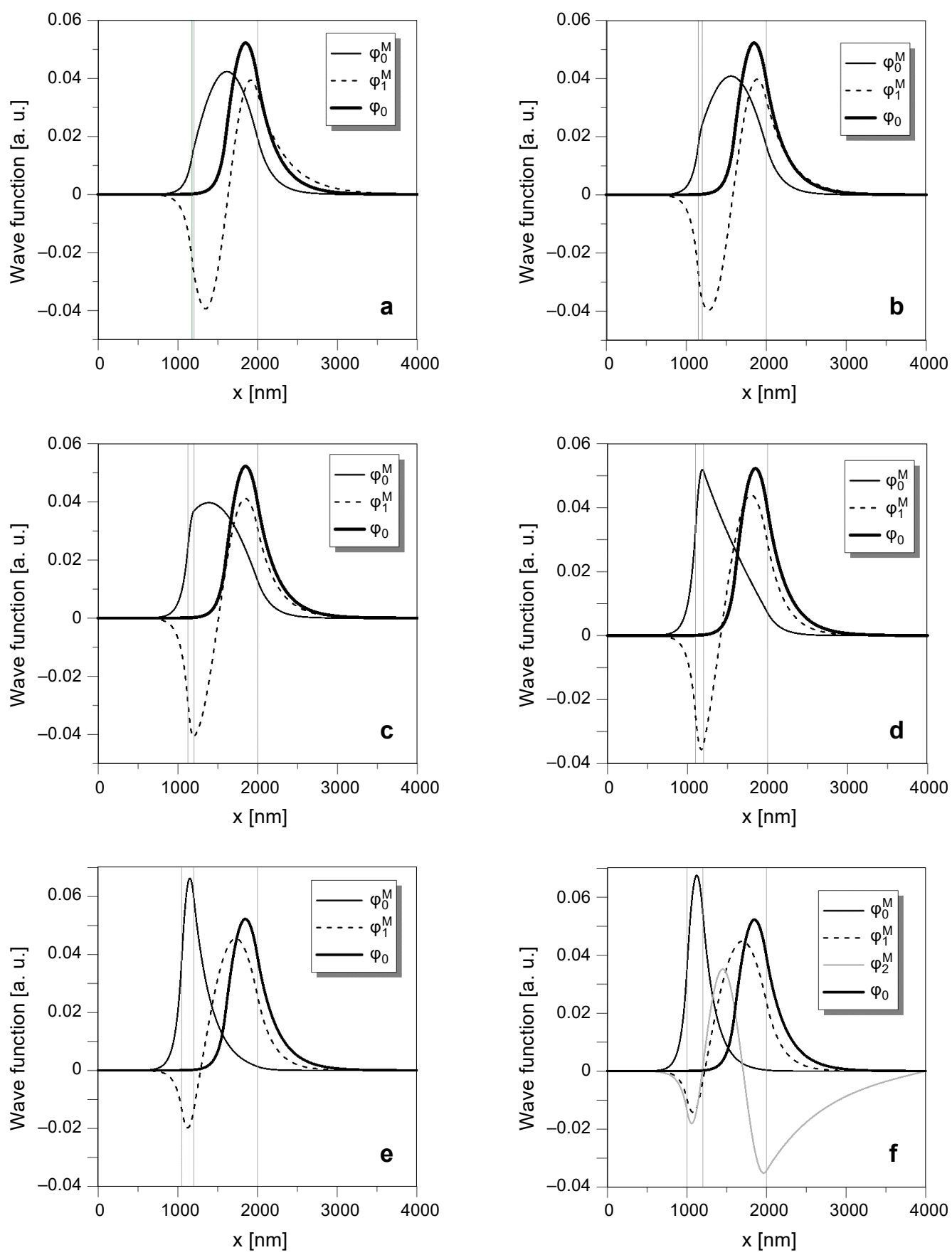

Fig. 2. The wave function $\varphi_{l}^{\mathrm{M}}$ distributions of multimode waveguide for the active nanometer layer of the thickness: $25 \mathrm{~nm}$ (a), $50 \mathrm{~nm}$ (b), $75 \mathrm{~nm}$ (c), $100 \mathrm{~nm}$ (d), $150 \mathrm{~nm}$ (e), and $200 \mathrm{~nm}$ (f) compared with wave function $\varphi_{0}$ distribution of the single-mode input/output waveguide. 
output waveguide. Vertical lines describe the multimode waveguide dimension and the sensor layer thickness.

The field at the distance $z$ is a superposition of all modal fields with different phase shifts:

$$
E_{\mathrm{M}}(x, z)=\sum_{l=0}^{N-1} c_{l} \varphi_{l}^{\mathrm{M}}(x) \exp \left(-j \beta_{l} z\right)
$$

Each mode of multimode waveguide propagates with different phase velocity and hence an interference pattern is produced along the waveguide section.

In the next step of the propagation, the field from the end of MMI section excites the output single-mode waveguide. The field at the output of the analyzed structure $E_{\text {out }}$ can be expressed by:

$$
E_{\text {out }}=c_{0} \varphi_{0}(x) \exp \left(-j \beta_{0} z\right)
$$

where $\beta_{0}$ is the propagation constant of single-mode waveguide and $c_{0}$ describes the overlap integral:

$$
c_{0}=\int_{-\infty}^{+\infty} E_{\mathrm{M}}\left(x, L_{\mathrm{MMI}}\right) \varphi_{0}(x) \mathrm{d} x
$$

where $L_{\mathrm{MMI}}$ is the multimode section length.

Taking into account Eqs. (5)-(7), the output power $P_{\text {out }}$ can be easily determined:

$$
P_{\text {out }} \sim\left|c_{0}\right|^{2}=\left|\int_{-\infty}^{+\infty} \varphi_{0}^{*}(x) \sum_{l=0}^{N-1} \varphi_{l}^{\mathrm{M}}(x) \exp \left(-j \beta_{l} L_{\mathrm{MMI}}\right) \mathrm{d} x\right|^{2}
$$

\section{Output signal analysis}

Power at the output of the sensor is described by Eq. (8). This dependence has been examined for different sensor layer geometry and its refractive index. In order to facilitate further discussion, phase shifts of modes at the end of the sensor are expressed in relation to the phase shift of the highest order mode,

$$
\begin{aligned}
P_{\text {out }} & \sim\left|c_{0}\right|^{2} \\
& =\left|\exp \left(-j \beta_{N-1} L_{\mathrm{MMI}}\right) \int_{-\infty}^{+\infty} \varphi_{0}^{*}(x) \sum_{l=0}^{N-1} c_{l} \varphi_{l}^{\mathrm{M}}(x) \exp \left[-j\left(\beta_{l}-\beta_{N-1}\right) L_{\mathrm{MMI}}\right] \mathrm{d} x\right|^{2} \\
& =\left|\int_{-\infty}^{+\infty} \varphi_{0}^{*}(x) \sum_{l=0}^{N-1} c_{l} \varphi_{l}^{\mathrm{M}}(x) \exp \left[j\left(\beta_{l}-\beta_{N-1}\right) L_{\mathrm{MMI}}\right] \mathrm{d} x\right|^{2}
\end{aligned}
$$


Let us introduce to Eq. (9) the beating length $(\mathrm{BL})$ of $(n, m)$ modes, defined as:

$$
L_{n, m}=\frac{2 \pi}{\beta_{n}-\beta_{m}}, \quad n<m
$$

on the base of it, the output signal can be determined by the beating lengths of several pairs of modes:

$$
\begin{aligned}
P_{\text {out }} & =\left|\int_{-\infty}^{+\infty} \varphi_{0}^{*}(x)\left[c_{N-1} \varphi_{N-1}^{\mathrm{M}}(x)+\sum_{l=0}^{N-2} c_{l} \varphi_{l}^{M}(x) \exp \left(\frac{j 2 \pi L_{\mathrm{MMI}}}{L_{l, N-1}}\right)\right] \mathrm{d} x\right|^{2} \\
& =\left|\int_{-\infty}^{+\infty} \varphi_{0}^{*}(x)\left[c_{N-1} \varphi_{N-1}^{\mathrm{M}}(x)+\sum_{l=0}^{N-2} c_{l} \varphi_{l}^{M}(x) \exp \left(j 2 \pi R_{l, N-1}\right)\right] \mathrm{d} x\right|^{2}
\end{aligned}
$$

where

$$
R_{l, N-1}=\frac{L_{\mathrm{MMI}}}{L_{l, N-1}}
$$

is the beating length number of $(l, N-1)$ modes.

The coefficient $R_{l, N-1}$ determines the number of the $\mathrm{BL}$ of $(l, N-1)$ modes in the MMI section length. This number depends on the refractive index changes of the active layer. On the base of the experimental results of optical properties of the $\mathrm{WO}_{3}$ nanolayer from the paper [9], it is assumed that the refractive index of the active layer has to be changed within the limit 1.900-1.905. Because the beating length number is huge for the considered length of the multimode section, the relative coefficient of BL number:

$$
\Delta R_{l, N-1}\left(n_{\mathrm{s}}\right)=R_{l, N-1}\left(n_{\mathrm{s}}\right)-R_{l, N-1}(1.9)
$$

is introduced to describe the output power dependence on refractive index changes of the active sensor layer. The output signal as a function of an active layer thickness is discussed for different limits of $\Delta R_{l, N-1}$.

\subsection{The case of $\Delta R_{l, N-1}\left(n_{\mathrm{s}}\right)<0$}

First, the active layer thickness within the limit of $20-83 \mathrm{~nm}$ is considered. It is the case of two-mode MMI section, which is determined by the $\Delta R_{01}$ relative coefficient of $\mathrm{BL}$ number. As can be seen from Fig. 3, the relative coefficient $\Delta R_{01}$ has a negative value in that range of layer thickness, which means that the beating length number decreases with increasing refractive index.

The relative coefficient of $\mathrm{BL}$ number $\Delta R_{01}\left(n_{\mathrm{s}}\right)$ decreases quickly with the increase of the refractive index, reaching the minimum value for the layer thickness near $60 \mathrm{~nm}$ 


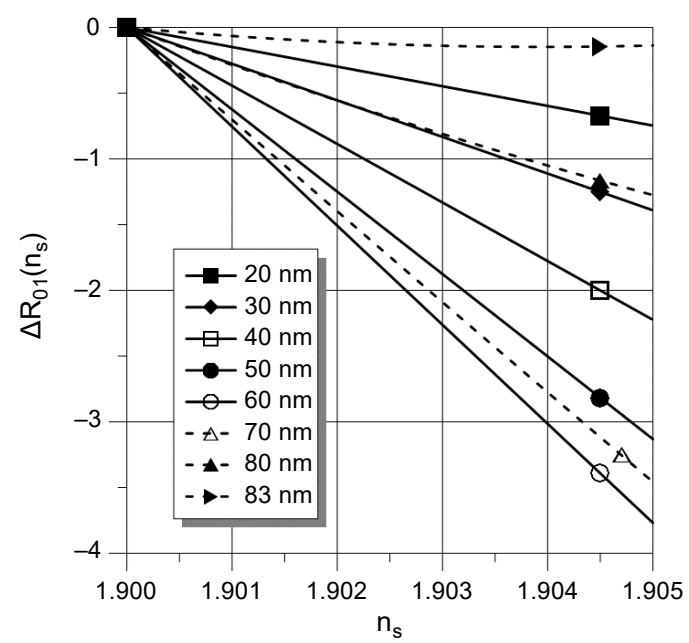

Fig. 3. Relative $\Delta R_{01}$ coefficient as a function of the refractive index of the active layer for different layer thicknesses in the limit $20-83 \mathrm{~nm}$.

(solid lines) and then it grows fast to the values close to zero for the nanolayer thickness near $85 \mathrm{~nm}$ (dashed lines).

The rate of $\Delta R_{01}$ variations influences the measurement range and sensitivity of the sensor. The sensitivity $S$ characterizes the rate of normalized output power $P_{\text {out }}$ changes with the refractive index of the sensor layer variations according to the expression:

$$
S=\frac{\partial P_{\text {out }}}{\partial n_{\mathrm{c}}}
$$

The unit of such defined sensitivity is 1/RIU (RIU - refractive index unit). In Fig. 4, output powers and sensitivities of the structures are presented as a function of the re-
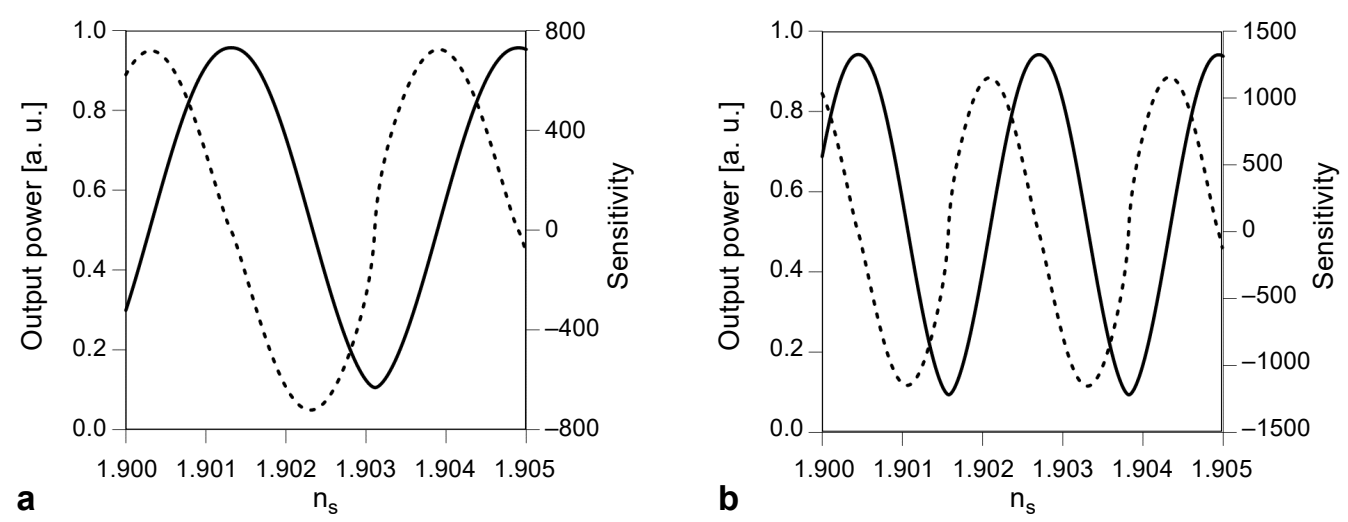

Fig. 4. The out power (solid line) and sensitivity (dashed line) as a function of the refractive index of the active layer, for the different layer thickness: $30 \mathrm{~nm}(\mathbf{a}), 40 \mathrm{~nm}(\mathbf{b}), 50 \mathrm{~nm}(\mathbf{c}), 60 \mathrm{~nm}(\mathbf{d}), 75 \mathrm{~nm}(\mathbf{e})$, and $80 \mathrm{~nm}(\mathbf{f})$. 

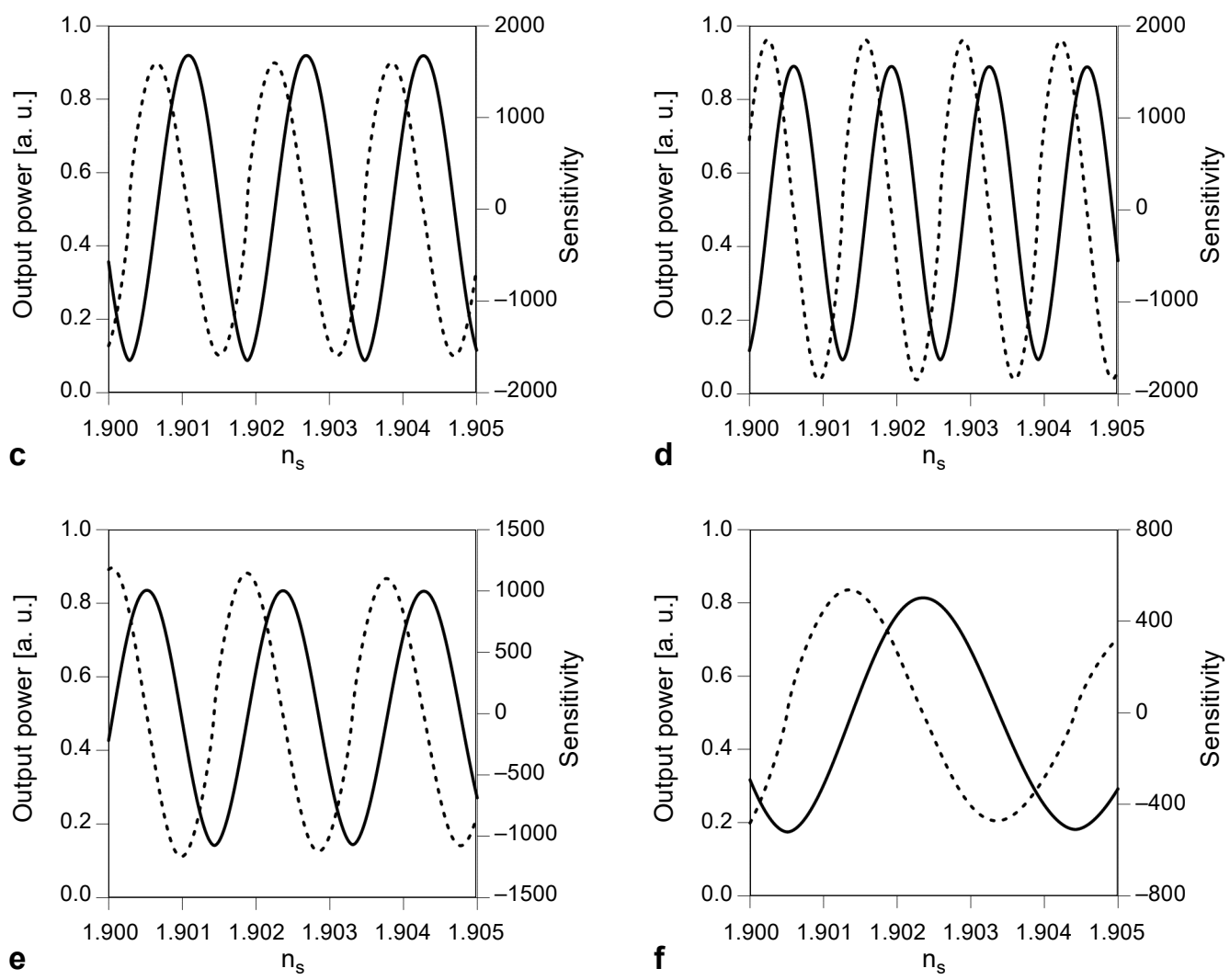

Fig. 4. Continued.

fractive index of the active layer, for different nanolayer thickness. One can see that the rate of $P_{\text {out }}$ changes increases with the layer thickness reaching the maximum at the value about $60 \mathrm{~nm}$ and then it falls similarly as the $\Delta R_{01}$ variations.

The range of $P_{\text {out }}$ variation is almost constant in the thickness limit from 20 to $60 \mathrm{~nm}$, which means that amplitudes of modes and excitation coefficients do not change with the refractive index of the nanolayer. Then it decreases slightly because the $\varphi_{0}^{\mathrm{M}}$ wave function, as can be seen from Fig. 2, moves towards the sensor layer, decreasing $c_{0}$ excitations coefficient.

Figure 5 presents the maximum value of the sensitivity $S_{\max }$ as a function of the active layer thickness. The function achieves maximum at $60 \mathrm{~nm}$.

Due to the periodic nature of the output signals, only the parts of the presented operating characteristics can be used for sensor applications, depending on the assumed resolution and the measurement range of the output signal. The examples of such dependences shown in Figs. $6 \mathbf{a}$ and $6 \mathbf{b}$ are limited to the range of refractive indices of the active layer $\Delta n_{\mathrm{s}}$ for which the sensitivity values reach $20 \%$ of the maximum value $S_{\max }$. 


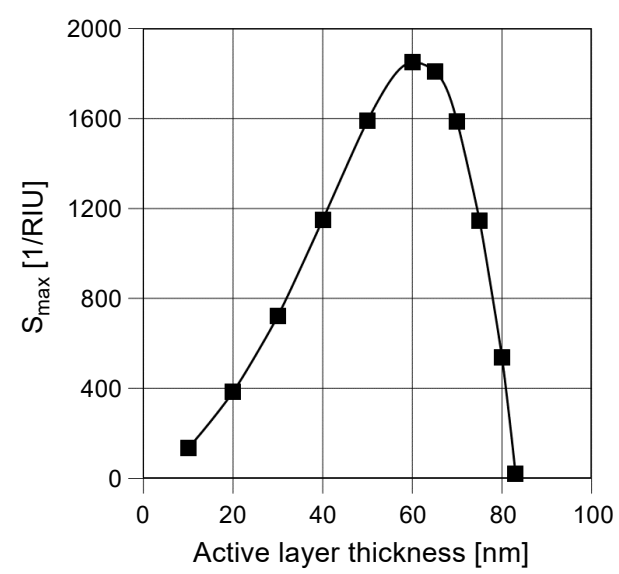

Fig. 5. Maximum value of the sensitivity $S_{\max }$ as a function of the active layer thickness.
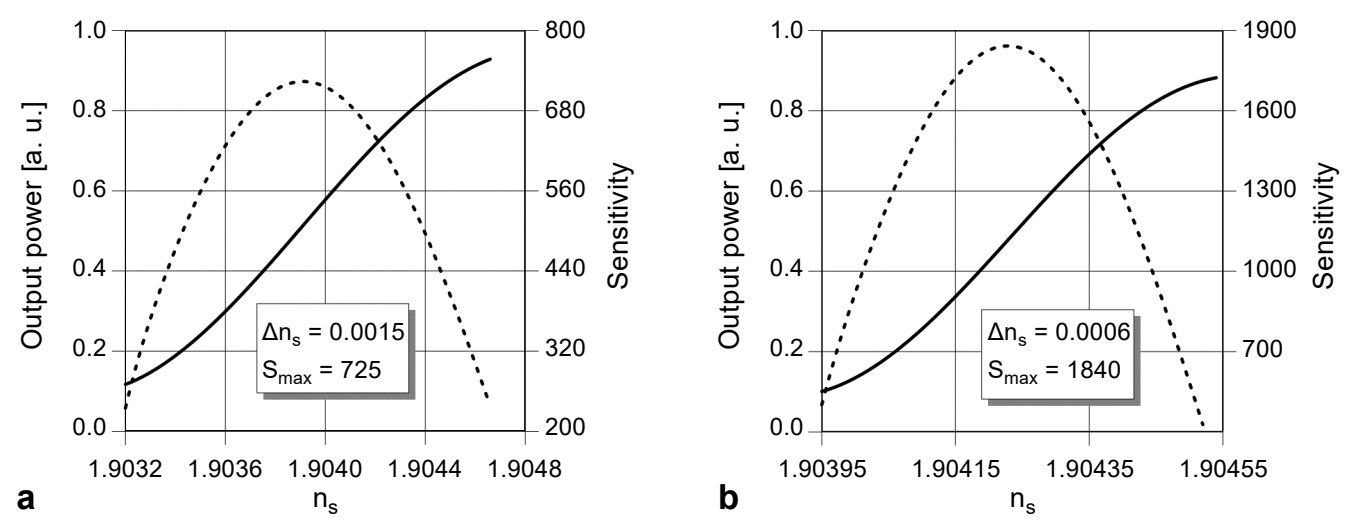

Fig. 6. The out power (solid line) and sensitivity (dashed line) for the layer thicknesses $30 \mathrm{~nm}$ (a) and $60 \mathrm{~nm}$ (b) as a function of the refractive index of the active layer, within the limits for which the sensitivity values reach $20 \%$ of their maximum.

The characteristics show monotonous nearly linear growth and their measurement range $\Delta n_{\mathrm{s}}$ and sensitivity strongly depend on the layer thickness.

\subsection{The case of $\Delta R_{l, N-1}\left(n_{\mathrm{s}}\right)>0$}

Within the limit of the active layer thickness equal to $85-160 \mathrm{~nm}$, the MMI section is still two-mode and its properties can be determined by the $R_{01}$ coefficient. As can be seen from Fig. 7, in that range of layer thickness the difference coefficient $\Delta R_{01}$ is positive. The beating length number grows very fast with the increase of the refractive index.

It results from the course of wave functions in the analyzed range of nanolayer thickness, shown in Fig. 2. Wave functions move towards the active layer, increasing 


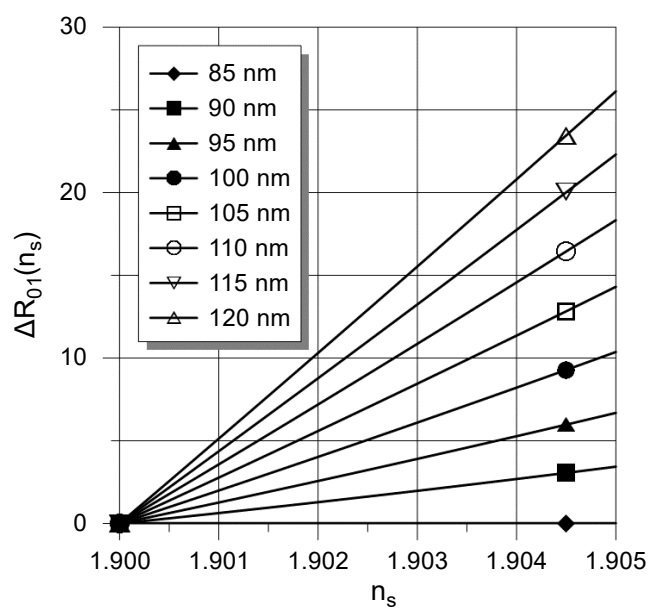

Fig. 7. Relative coefficient $\Delta R_{01}$ as a function of the refractive index of the active layer for different layer thicknesses.

its impact on the output power, especially for the nanolayer thickness above $100 \mathrm{~nm}$. Field distribution of wave function $\varphi_{0}^{\mathrm{M}}$ of the fundamental mode then concentrates very close to a nanolayer of a high refractive index, which increases its propagation constant. The propagation constant of the first order mode changes much slower.

Figure 8 shows the output power and sensitivity dependence on the refractive index of the active layer for different layer thicknesses. Due to the high rate of the output signal changes for the thicknesses from 110 to $150 \mathrm{~nm}$, the range of refractive index changes is restricted to the limit 1.900-1.902.

As can be seen from Fig. 9, the sensitivity, as in the previous case, strongly depends on the sensor layer thickness and it reaches the maximum for the layer thickness near $110 \mathrm{~nm}$.
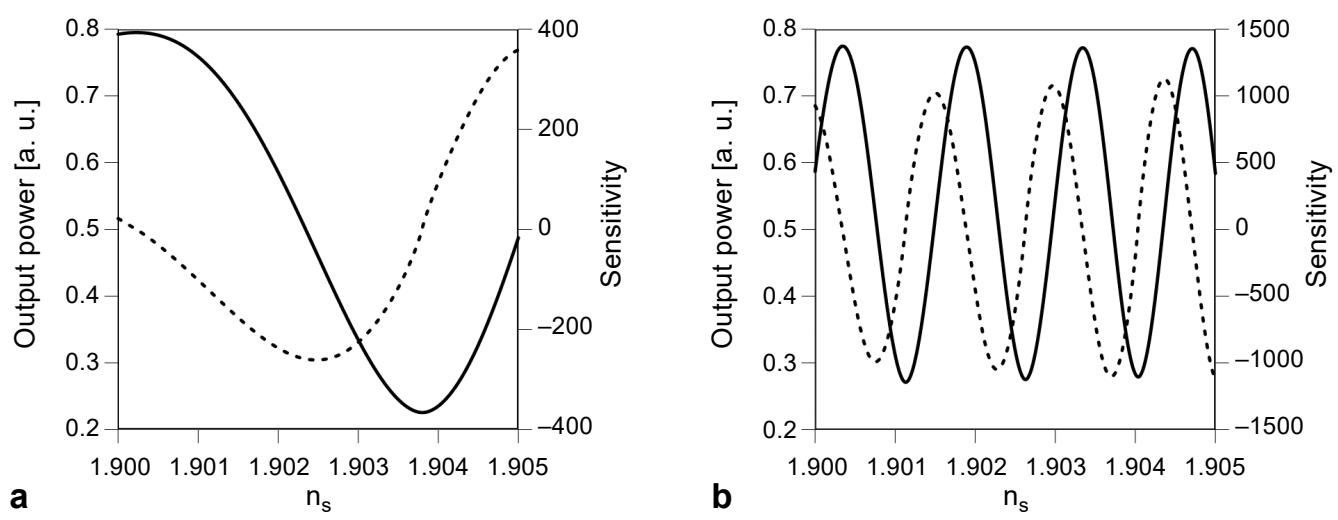

Fig. 8. The out power (solid line) and sensitivity (dashed line) as a function of the refractive index of the active layer, for different layer thickness: $85 \mathrm{~nm}(\mathbf{a}), 90 \mathrm{~nm}(\mathbf{b}), 100 \mathrm{~nm}(\mathbf{c}), 110 \mathrm{~nm}(\mathbf{d}), 120 \mathrm{~nm}(\mathbf{e})$, and $140 \mathrm{~nm}(\mathbf{f})$. 

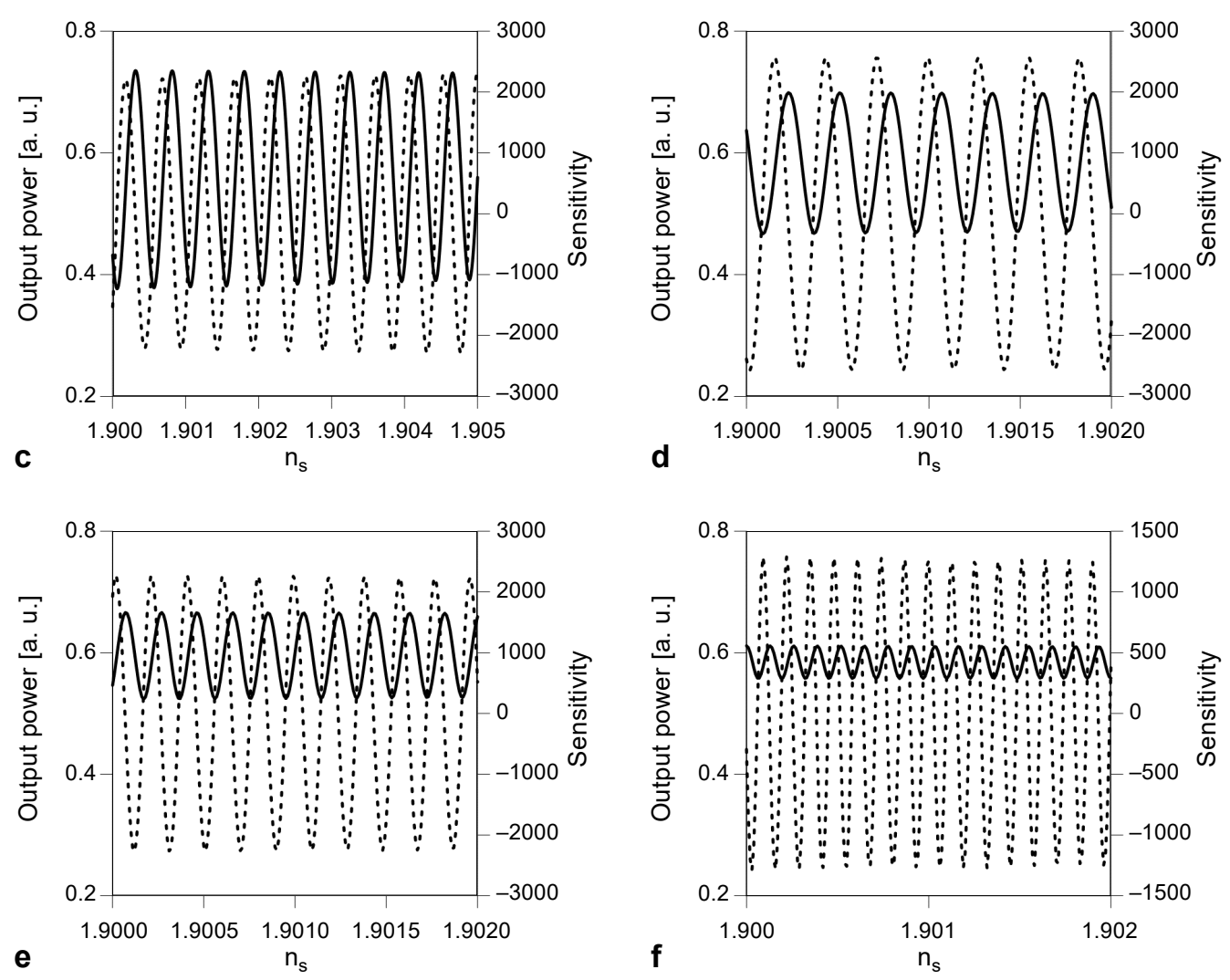

Fig. 8. Continued.

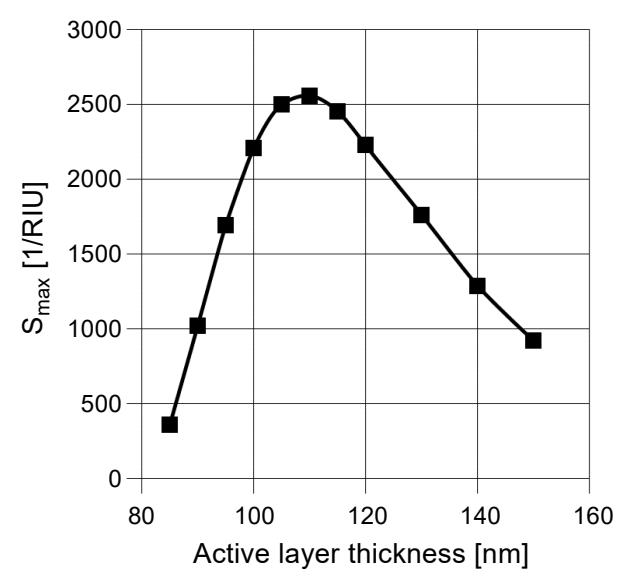

Fig. 9. The maximum value of the sensitivity $S_{\max }$ as a function of the active layer thickness for the range of $85-150 \mathrm{~nm}$. 
The characteristic feature of the presented output signals is the decreasing depth of the modulation. The range of output power variations decreases to the value below 0.1 . It is in close connection with wave function characteristics. The $\varphi_{0}^{\mathrm{M}}$ wave function moves towards the external surface with the increasing thickness of the sensor layer. Its distribution restricts to the area of the nanolayer and its vicinity. This effect decreases the overlap integral described by Eq. (3) and the value of $c_{0}$ excitation coefficient. The $\varphi_{l}^{\mathrm{M}}$ distribution changes to a lesser extent and the $c_{1}$ value only slightly increases. As can be seen from Fig. 10, the difference between the values $c_{0}$ and $c_{1}$ in-

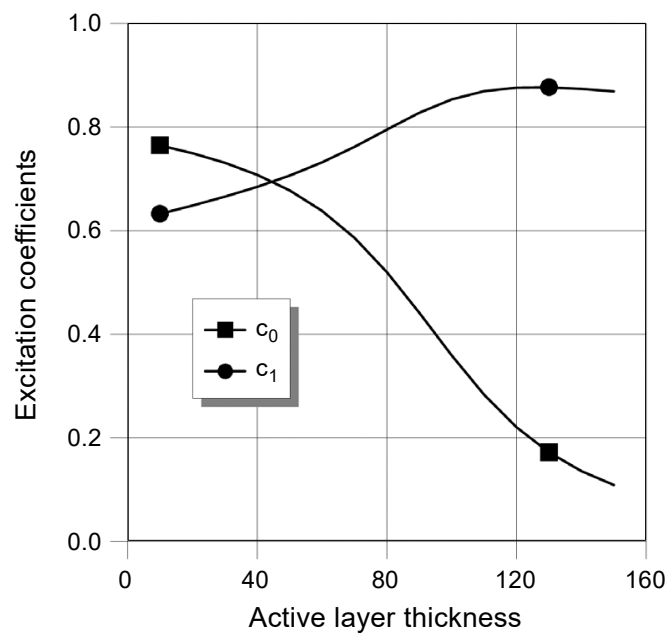

Fig. 10. Excitation coefficients $c_{0}$ and $c_{1}$ dependence on the active layer thickness.
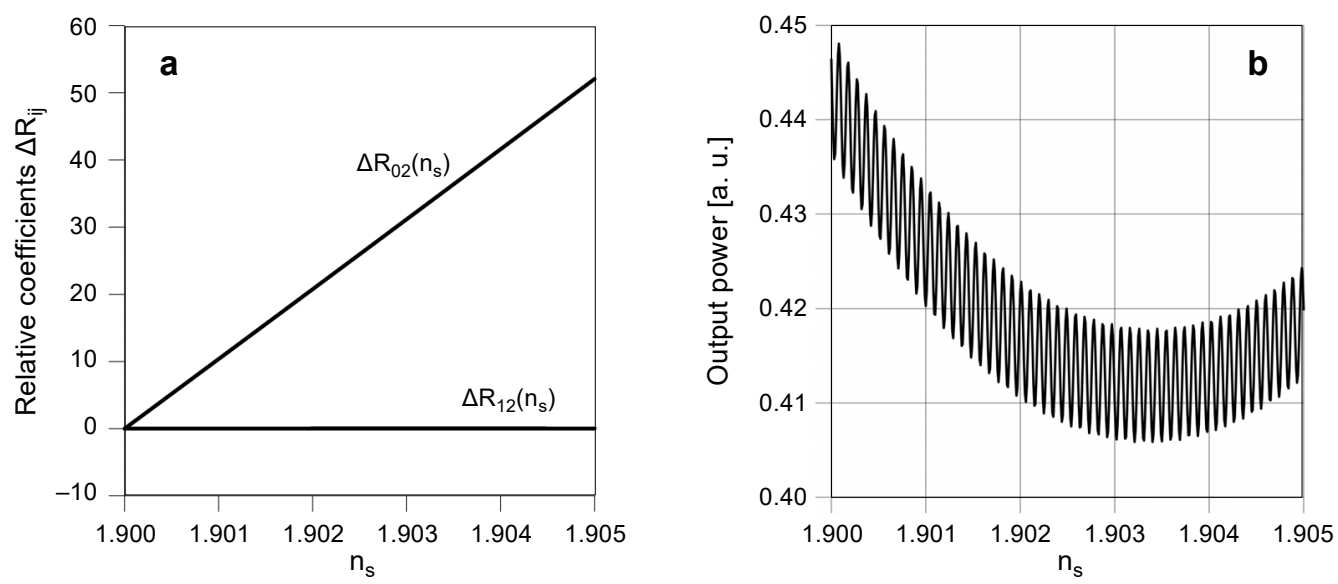

Fig. 11. Properties of three-mode MMI section with the active layer thickness amounts to $170 \mathrm{~nm}$ : $\Delta R_{12}$ and $\Delta R_{02}$ relative coefficients of $\mathrm{BL}$ number (a), and the output power (b) as a function of the nanolayer refractive index. 
creases with the active layer thickness and the interfering modes have significantly different amplitudes. It affects the depth of signal modulation.

\subsection{The case of the three-mode MMI section}

The MMI section is three-mode for the active layer thickness over $165 \mathrm{~nm}$, and the $R_{02}$ and $R_{12} \mathrm{BL}$ number coefficients determine its properties. Figure 11 presents the properties of the structure with the $170 \mathrm{~nm}$ active layer. The $\Delta R_{12}$ and $\Delta R_{02}$ relative coefficients of $\mathrm{BL}$ number have entirely different ranges of variations $-\Delta R_{12}$ are very close to zero within the limit $(0,0.02)$ and $\Delta R_{02}$ changes from 0 to 52 . The output power is for that reasons the composition of fast varying function connected with $\Delta R_{02}$, with a small amplitude of modulation, which amounts to 0.1 and a slowly varying envelope connected with $\Delta R_{12}$. The optical structure is characterized by a small range of the output signal variations and little sensitivity whose maximum value amounts to 400 . It is caused by significant mismatching of the $\varphi_{0}^{\mathrm{M}}$ distribution to the single-mode input and output wave function.

\section{Conclusions}

The work presents detailed examinations of a one-dimensional multimode waveguide structure with a thin active sensor nanolayer, which properties correspond to $\mathrm{WO}_{3}$, a promising material for the detection of several gas types. It is assumed that the refractive index of the layer is about 1.9 and its depth changes in the limit from 0 to $200 \mathrm{~nm}$. The results show the strong dependence of the examined structure properties on the nanolayer thickness. The number of the beat length of modes in the MMI section length and excitations coefficients of modes determines the output characteristics of the sensor.

For the small thicknesses of the active layer, for the assumed material parameters within the limit of 20-85 nm, MMI section is two-mode. The beat length of modes in the MMI section length decreases with the refractive index of the active layer, and the excitations coefficients change slightly. The sensitivity strongly depends on the layer thickness, achieving maximum at $60 \mathrm{~nm}$. The range of output power variations is almost constant in this thickness limit. From the characteristics, it is possible to determine the refractive index variations of the sensor nanolayer below $10^{-5}$.

For the larger thicknesses of the active layer, the MMI section is still two-mode, but the beat length of modes in the MMI section length increases with the refractive index of the active layer. The difference between the values of excitations coefficients increases with the active layer thickness and the interfering modes have significantly different amplitudes. It affects the depth of signal modulation, which tends to 0.1.

The MMI section is three-mode for the active layer thickness over $165 \mathrm{~nm}$. The optical structure is characterized by a small range of the output signal variations and a little sensitivity. It is connected with the significant mismatching of the distribution of the 
fundamental mode of the multimode waveguide to the single-mode input and output wave function. The dynamic range and resolution of the signal restrict the possibility of sensor applications.

\section{References}

[1] Ramirez J.C., Lechuga L.M., Gabrielli L.H., Hernandez-Figueroa H.E., Study of a low-cost trimodal polymer waveguide for interferometric optical biosensors, Optics Express 23(9), 2015, pp. 11985 -11994, DOI: 10.1364/OE.23.011985.

[2] Mazingue T., Kribich R.K., Etienne P., Moreau Y., Simulations of refractive index variation in a multimode interference coupler: application to gas sensing, Optics Communications 278(2), 2007, pp. 312-316, DOI: 10.1016/j.optcom.2007.06.036.

[3] SzewczuK A., BŁahut M., Applications of gradient index multimode interference structures in the technology of optical sensor, Acta Physica Polonica A 120(4), 2011, pp. 740-743, DOI: 10.12693/ APhysPolA.120.740.

[4] SzewczuK A. BŁahut M., MMI structures covered by bromocresol purple for ammonia detection, Acta Physica Polonica A 124(3), 2013, pp. 570-573, DOI: 10.12693/APhysPolA.124.570.

[5] Gut K., Bimodal layers of the polymer SU8 as a refractometer, Procedia Engineering 47, 2012, pp. 326-329, DOI: 10.1016/j.proeng.2012.09.149.

[6] Nordström M., Zauner D., Boisen A., HÜBNER J., Single-mode waveguides with SU-8 polymer core and cladding for MOEMS applications, Journal of Lightwave Technology 25(5), 2007, pp. 1284-1289.

[7] Karouta F., Vora K., Tian J., Jagadish C., Structural, compositional and optical properties of PECVD silicon nitride layers, Journal of Physics D: Applied Physics 45(44), 2012, article 445301, DOI: $10.1088 / 0022-3727 / 45 / 44 / 445301$.

[8] Wang C., Yin L., Zhang L., Xiang D., Gao R., Metal oxide gas sensors: sensitivity and influencing factors, Sensors 10(3), 2010, pp. 2088-2106, DOI: 10.3390/s100302088.

[9] Von Rottkay K., Rubin M., Wen S., Optical indices of electrochromic tungsten oxide, Thin Solid Films 306(1), 1997, pp. 10-16, DOI: 10.1016/S0040-6090(97)00254-X.

[10] YANG M., Sun Y., Zhang D., JiAng D., Using $P d / W O_{3}$ composite thin films as sensing materials for optical fiber hydrogen sensors, Sensors and Actuators B: Chemical 143(2), 2010, pp. 750-753, DOI: $10.1016 /$ j.snb.2009.10.017.

[11] MACIAK E., Fiber optic sensor for $\mathrm{H}_{2}$ gas detection in the presence of methane based on $\mathrm{Pd} / W \mathrm{O}_{3}$ low-coherence interferometric structure, Proceedings of SPIE 10455, 2017, article 104550W, DOI: $10.1117 / 12.2282891$.

[12] Liu R., Lu B.-R., XIE S.-Q., Wan J., Shu Z., Qu X.-P., Chen Y., Optical nanostructures fabricated by $S U-8$ based nanoimprint lithography, Journal of the Korean Physical Society 55(3), 2009, pp. 1290-1294. 